\title{
Erratum: Electron induced molecular desorption from electron clouds at the Relativistic Heavy Ion Collider \\ [Phys. Rev. ST Accel. Beams 8, 113201 (2005)]
}

\author{
Ubaldo Iriso and Wolfram Fischer \\ (Received 16 January 2006; published 22 February 2006) \\ DOI: 10.1103/PhysRevSTAB.9.029901 \\ PACS numbers: 29.27.Bd, 68.47.-b, 07.30.Kf, 99.10.Cd
}

The present erratum adds some comments and further references to our earlier paper [1]. None of the comments in this Erratum affect the results and conclusions in the original manuscript.

(i) In the original manuscript the authors used Ref. [2] to explain the saturation of the electron cloud buildup. This reference states that a simple criterion for the importance of space charge forces is a Debye length of the electron cloud comparable to or smaller than the beam pipe radius. It has been pointed out to us [3] that the space charge effects were previously accounted for through electrostatic analysis and detailed simulation codes in other references, for example $[4,5]$. The common conclusion yields that the saturation of the electron density occurs at a value close to the neutralization level: the line density of the electron cloud is similar to the line density of the positively charged beam. Moreover, Ref. [5] shows the difference between a cloud buildup if self-cloud electric fields are taken into account (saturation) or not (indefinite growth).

(ii) Comparisons between measured and simulated spectrums were carried out previously in other machines. Good agreements were found in the Proton Storage Ring (PSR) at LANL [6], in the Super Proton Synchrotron (SPS) at CERN [7], and in the B-factory at KEKb [8].

(iii) The electron detector calibration shown in Fig. 15 is courtesy of He [9].

\section{ACKNOWLEDGMENTS}

We are grateful to F. Zimmermann (CERN) for helpful comments.

[1] Ubaldo Iriso and Wolfram Fischer, Phys. Rev. ST Accel. Beams 8, 113201 (2005).

[2] G. Stupakov, CERN-LHC-Project-Report-141, 1997.

[3] F. Zimmermann (private communication).

[4] S. Heifets, in Proceedings of ECLOUD'02, Geneva, Switzerland, 2002 edited by G. Rumolo and F. Zimmermann (CERN, Geneva, 2002).

[5] F. Zimmermann, CERN-LHC-Project-Report-95, 1997.

[6] M. Pivi and M. Furman, Phys. Rev. ST Accel. Beams 6, 034201 (2003).

[7] G. Arduini, V. Baglin, T. Bohl, B. Jenninger, J. M. Jimenez, J.-M. Laurent, F. Ruggiero, D. Schulte, and F. Zimmermann, in Proceedings of the European Particle Acceleerator Conference, Lucerne, Switzerland (CERN, Geneva, Switzerland, 2004).

[8] H. Fukuma, Proceedings of ECLOUD'02, Geneva, Switzerland, 2002 (Ref. [4]).

[9] P. He, H.C. Hseuh, D. Gassner, J. Gullotta, D. Trbojevic, and S. Y. Zhang, in Proceedings of the Particle Accelerator Conference, Portland, OR, 2003 (IEEE, Piscataway, NJ, 2003). 